\title{
Tratamiento de la muñeca inestable en la parálisis cerebral
}

\author{
J. Minguella ${ }^{(1)}$, L. Terricabras ${ }^{(2)}$, M. Cabrera $^{(2)}$ \\ ${ }^{(1)}$ EX JEFE DEL SERVICIO. \\ ${ }^{(2)}$ MÉDICO ADJUNTO. \\ Servicio de Cirugía Ortopédica Infantil. Hospital Universitario Sant Joan de Déu, Barcelona. \\ CENTRO MÉdico TEKNon, BARCELONA.
}

\author{
Correspondencia: \\ Dr. Joan Minguella \\ C/. Temple, 24, $2^{\text {o }}$ \\ 08911 Badalona \\ Tfnos.: 933893159 (particular) y 933933129 (consultorio C. M. Teknon) \\ Fax: 933933030 \\ E-mail: 2809jms@comb.es
}

\begin{abstract}
Estudio de los resultados obtenidos con el tratamiento quirúrgico de la mano en 22 pacientes (23 manos) afectos de parálisis cerebral. En 16 manos se trasplantó el cubital anterior a los tendones extensores radiales, y en 7 manos se realizó una artrodesis de muñeca. Se practicó una aponeurotomía de la musculatura epitroclear en 8 manos además del trasplante de cubital anterior, y en 3 junto con la artrodesis. Un dato pronóstico significativo fue la extensión activa de los dedos: el 84,6\% de estas manos ganaron función. Mejoraron la función todas las manos que previamente eran ya algo funcionales. Todas las manos mejoraron también su posición y aspecto; aparte la estética, la buena posición favorece la utilidad de la mano. En las artrodesis se resecó la hilera proximal del carpo y la prominencia del hueso grande se introdujo en la epífisis radial, consiguiendo con ello una mayor estabilidad.
\end{abstract}

A study of the results obtained with the surgical treatment of the hand in 22 patients ( 23 hands) suffering from cerebral palsy. In 16 hands the flexor carpi ulnaris was transplanted to the radial extensor tendons, and in 7 hands, a wrist arthrodesis was performed. An aponeurotomy of the epitroclear musculature was carried out on 8 hands as well as the carpi ulnaris transplant, and in 3 together with the arthrodesis. A significant result was the active extension of the fingers: $84.6 \%$ of the hands became functional. There was an improvement in function in all hands that were previously somewhat functional. All the hands also improved their position and appearance; apart from the aesthetic factor, a good position enhances the use of the hand. In the artrodeses, the carpal proximal row was dried, and the prominence of the large bone was introduced into the radial epiphysis, which produced improved stability.

Rev. Iberam. Cir. Mano - Vol. 32 • Núm. 65 - Octubre 2004 (24-28)

\section{INTRODUCCIÓN}

$\mathrm{N}$ o es fácil la decisión de tratar quirúrgicamente la mano de un niño con parálisis cerebral. Globalmente habría que distinguir dos tipos de problema en estos pacientes:

1) En unos casos la problemática reside en la posición de la mano, con la muñeca en fle- xión palmar y los dedos en flexión irreductible, o en extensión, y además un componente de pronación; todo ello conlleva un problema estético, de higiene de la mano (a veces con lesiones de maceración cutánea en la palma) y dificultad en vestir un paciente con la mano en esta posición. En estos casos la falta de función no suele ser el motivo de la consulta. 
2) Pacientes con una situación semejante, pero menos acusada, y en la que se valora la falta de función que ello representa; es una mano con un cierto control que se pretende mejorar.

Si bien el nivel intelectual del paciente tiene valor en este último caso, porque representará una colaboración que puede ser eficaz, los planteamientos ante un paciente del primer grupo pueden prescindir de este aspecto, aunque en ocasiones nos puede sorprender una mejoría funcional no esperada.

En ambos casos la actuación quirúrgica fundamental es la estabilización de la muñeca, con la que mejora la posición y aspecto de la mano, y en los casos funcionales ofrece unas mejores condiciones para la actuación de los flexores y extensores de los dedos. Sakellarides, H. T. y Kirvin, F. M. (1995) llegan a la conclusión de que, en la parálisis cerebral, la presencia de una muñeca con desequilibrio muscular no compensado, es uno de los problemas más incapacitantes, y limita la función de la mano en un $60 \%$.

El objetivo de este trabajo es estudiar los resultados que se han obtenido con el tratamiento de estas manos.

\section{MATERIAL Y MÉTODO}

Se han revisado las Historias Cínicas de 22 pacientes (12 varones y 10 niñas), con 23 manos tratadas quirúrgicamente. Son casos afectados de parálisis cerebral infantil de origen conatal, y no están incluidos pacientes de otras etiologías; 21 eran espásticos y un caso atetósico. De estos pacientes 15 presentaban hemiplejía y 7 tetraplejía. Si bien el tratamiento de estas manos, en general, se limita a las hemiplejías, se ha extendido a las tetraplejías con afectación claramente asimétrica, en que se podía mejorar una mano, o cuando la posición de la mano representaba un problema para la higiene o dificultad para vestirse. En un caso se operaron ambas manos; después de operar la peor, ésta pasó a ser la predominante y solicitaron operar la otra.

La valoración preoperatoria se simplificó a la exploración de los siguientes parámetros: 1) Función de la mano; más que grados de fun- ción se ha valorado si el paciente utilizaba o no la mano, aunque fuera como simple ayuda de la mano sana, y en el posoperatorio se ha tenido en cuenta si, según los padres, el paciente utilizaba la mano más o mejor que antes. 2) Capacidad o no de extender los dedos activamente (Figura 1), aunque precisara una flexión de la muñeca. 3) Grado de flexión en que mantenía la muñeca, y 4) Si había problemas en cuanto a la higiene de la mano, y si la posición dificultaba vestir al paciente.

Se han utilizado fundamentalmente dos técnicas:

1) Trasplante del cubital anterior (flexor carpi ulnaris) a dorso de la muñeca, suturando el tendón a un radial o, en ocasiones, a ambos (extensor carpi radialis brevis o longus). El objetivo era suprimir la principal fuerza deformante de la muñeca, proporcionar una extensión activa y a la vez conseguir una cierta supinación, favorecida por el trayecto que sigue el tendón del cubital anterior, dando la vuelta sobre el cúbito, para alcanzar el dorso de la mano. Se ha procurado que la tensión de la sutura mantenga pasivamente la muñeca en extensión neutra. Se ha practicado esta técnica en 16 manos, a una edad entre 7 años 4 meses y 18 años 4 meses, con una edad media de 12 años 8 meses. En 8 de estas manos no se apreciaba función alguna, en 7 era escasa, y una mano tenía una función eficaz. En 11 manos se conseguía una extensión activa de los dedos con la muñeca en flexión.

2) Artrodesis de la muñeca, que se ha practicado con resección de la hilera proximal del carpo, a fin de relajar la tensión palmar. Se re-

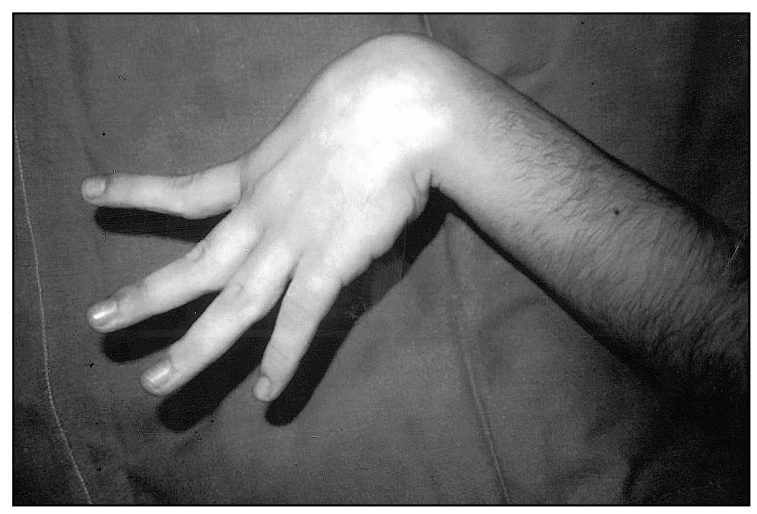

Figura 1. La mano es capaz de extender los dedos con la muñeca en flexión. 


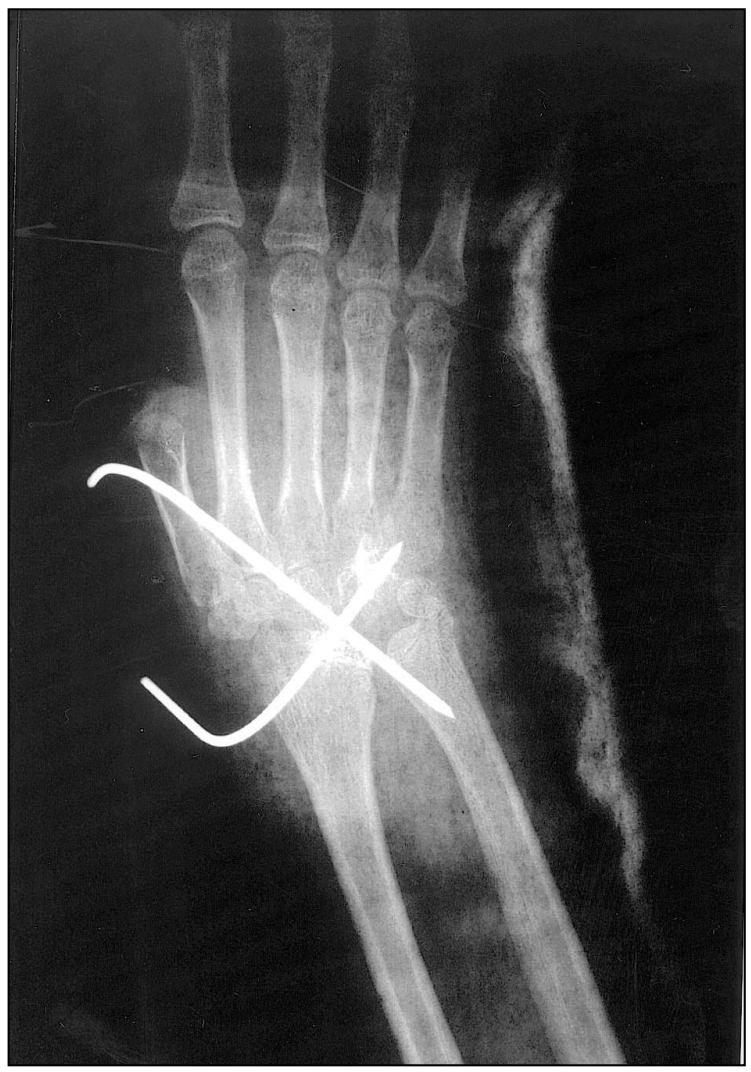

Figura 2. Radiografía que muestra la muñeca después de practicar la artrodesis, con resección de la hilera proximal del carpo, y fijación con dos agujas de Kirschner.

seca el cartílago articular del radio y del carpo, dejando la prominencia del hueso grande (os capitate), que al extender la muñeca se introduce dentro de un orificio previamente practicado en la epífisis distal del radio, con lo que se favorece la fusión (Minguella, J., 1979). Se mantiene la posición con unas agujas de Kirschner o con unas grapas, si se ha agotado ya la fisis del radio (Figura 2). La posición ideal es de una extensión neutra y discreta inclinación cubital; sin embargo cuando debe utilizarse una silla de ruedas o cuando esta mano debería utilizarse para la higiene personal, es mejor dar un poco de flexión palmar, de unos $20^{\circ}$. Se ha realizado la artrodesis de muñeca en 7 manos, una de ellas atetósica, y a una edad entre 11 años y 16 años 9 meses, con una edad media de 15 años 4 meses. Ninguna de estas manos era funcional y sólo 2 eran capaces de extender activamente los dedos con la muñeca en flexión.

Ambas técnicas se han combinado con una aponeurotomía de la musculatura epitroclear, cuando lo aconsejaba la excesiva flexión de los dedos, con la muñeca en extensión. En los casos de trasplante del cubital anterior se ha evitado la desinserción de este músculo de la epitróclea, con el fin de no debilitar su potencia de contracción. Se ha actuado sobre la musculatura epitroclear en 11 manos, 8 de ellas combinadas con el trasplante del cubital anterior y en 3 con una artrodesis de la muñeca.

En 7 manos se precisó alguna actuación complementaria sobre partes blandas para mejorar la posición del pulgar.

Se indicó el trasplante del cubital anterior cuando la mano tenía alguna función o un cierto control de la extensión activa de los dedos, aun teniendo que flexionar la muñeca. Se indicó la artrodesis ante una contractura en flexión de la muñeca de más de $60^{\circ}$, que no podría corregir una cirugía sobre partes blandas, y en manos sin ninguna función en pacientes con nivel intelectual bajo; es la única técnica aconsejable en pacientes con atetosis. Sin embargo es difícil definir con exactitud las indicaciones; en ocasiones se ha colocado una férula de yeso para observar el comportamiento funcional de flexión-extensión de los dedos y capacidad de presa con la muñeca estable, que nos ayudara a decidir.

\section{RESULTADOS}

De las 16 manos en que se practicó un trasplante del cubital anterior, 15 mejoraron su posición $\mathrm{y}$ aspecto y una sólo de manera parcial. Esta mano, en el preoperatorio no tenía extensión activa de los dedos y la muñeca estaba en flexión de $90^{\circ}$; probablemente fue una mala indicación y hubiera precisado una artrodesis, que es lo que después se le propuso. Las manos que previamente tenían una cierta función, todas la mejoraron (Figura 3), y de las inactivas, 2 adquirieron función después del tratamiento quirúrgico; una de ellas en la exploración preoperatoria era capaz de extender activamente los dedos.

Las 7 manos en las que se practicó una artrodesis, todas mejoraron la posición y el aspecto, y 3 adquirieron una cierta función, que antes no tenían; dos de estas manos en el pre- 

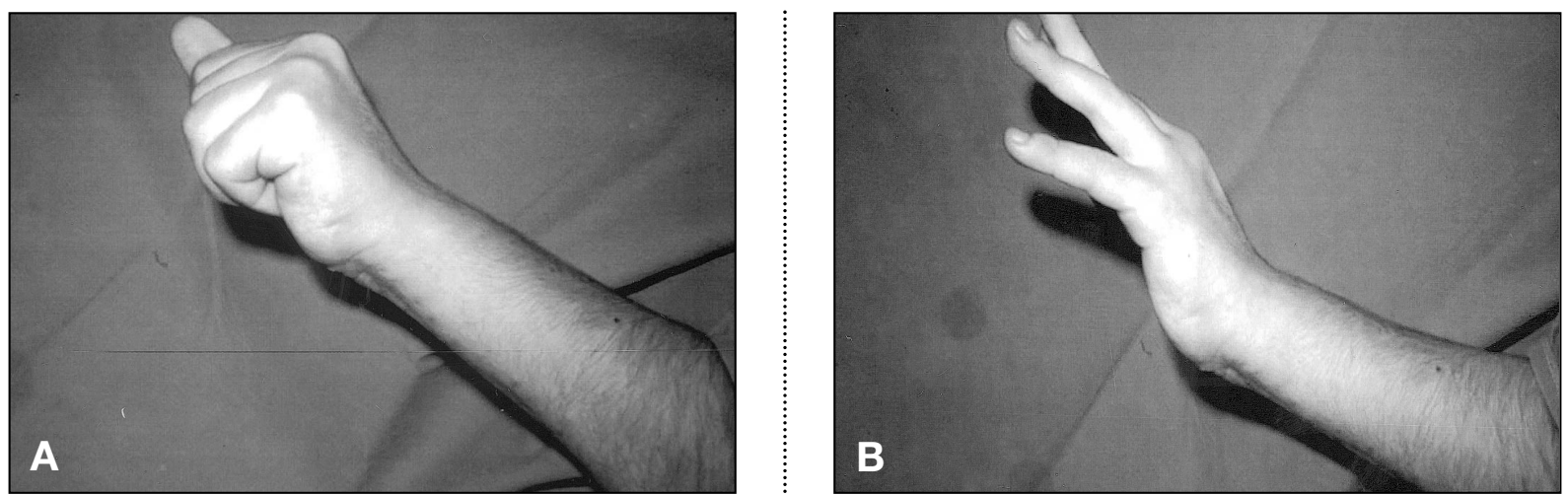

Figura 3. Resultado de la mano de la Figura 1 después del trasplante del cubital anterior a los tendones extensores radiales: A) con la muñeca en extensión activa, puede flexionar los dedos, y B) también extenderlos, aunque de forma incompleta.

operatorio eran capaces de extender activamente los dedos con la muñeca en flexión. En un caso hubo un retraso en la consolidación de la artrodesis, que se solucionó prolongando la inmovilización.

De las 23 manos estudiadas, 13 extendían activamente los dedos en la exploración preoperatoria, y de ellas $11(84,6 \%)$ ganaron función. De las 10 manos sin extensión activa previa de los dedos, sólo 2 (20\%) ganaron función.

Los resultados son difíciles de valorar, en cuanto a la función de la mano, ya que el hecho de que en la consulta el paciente sea capaz de coger un objeto cuando se le pide, no quiere decir que utilice luego esta función. En ocasiones, en posteriores exploraciones se constata que si bien el aspecto de la mano es mucho mejor, en ningún momento el paciente utiliza esta mano de modo espontáneo y se olvida de la función que parecía haber obtenido.

\section{DISCUSIÓN}

Las funciones de la mano se adquieren con una cierta facilidad hasta los 3 años de edad (se ha podido comprobar en el tratamiento de las malformaciones de la mano), pero a partir de aquí cada vez se hace más difícil adquirir una nueva función, y esta dificultad, si cabe, es aún mayor con una capacidad intelectual más bien limitada, como es el caso de los niños con parálisis cerebral. Es por este motivo que si bien al principio, probablemente por el estímulo de la rehabilitación, una mano operada es capaz de coger un objeto, esta función puede no asumirse y es probable no consiga utilizarla de manera espontánea; con el tiempo la función se olvidará. Hay que distinguir, pues, entre lo que el niño puede hacer y lo que realmente hace. Esto nos ha hecho pensar que quizás habría que realizar los trasplantes tendinosos más precozmente, aunque algunos autores (Beach, W. R., et al., 1991) manifiestan que no han observado grandes diferencias relacionadas con la edad de la operación.

En general no hay que esperar la aparición de una función nueva, aunque sí la mejoría de la función que ya tenía. Los resultados de nuestro estudio muestran que sólo han mejorado la función las manos que previamente eran ya algo funcionales, es decir, que la función estaba ya presente en el esquema cerebral del paciente, y esto se confirma también en otros trabajos (Alexander, R. D. et al., 2000; Beach, W. R. et al., 1991) que postulan que el elemento pronóstico más significativo es un cierto control voluntario de la mano y que se utilice, aunque sólo sea como ayuda a la mano sana. Pero siempre cabe la posibilidad que en alguna ocasión, como se ha podido constatar en nuestros casos, y también en la literatura (Hoffer, M. M. y Zeitzew, S., 1988; Rayan, G. M. y Young, B.T., 1999), se produzca una cierta mejoría de la función no prevista, dado el estado inicial de esta mano.

Sin embargo, no hay que infravalorar el hecho de que se consiga sólo una mejor posición, ya que ello facilitará también la utilidad de la mano (Beach, W. R. et al., 1991) al actuar me- 
jor su musculatura con la muñeca estable. Al disminuir la pronación, permite oponer las palmas de ambas manos para coger un objeto (Wolf, T. M. et al., 1998) y una mejor posición favorece además la integración social del paciente (Rayan, G. M. y Young, B. T., 1999) y una mejor calidad de vida, sin el estigma de una mano con evidencia de anormalidad.

En los resultados obtenidos cabe considerar una cierta relación entre la adquisición de función y la capacidad previa de extender activamente los dedos, aun sin una función constatable; así, el 84,6\% de manos con extensión activa de los dedos ganaron función, en contraste con sólo el $20 \%$ de las que no tenían extensión activa. Ello concuerda con las conclusiones de otros trabajos (Wenner, S. M. y Johnson, K. A., 1988; Wolf, T. M. et al., 1998) en que se manifiesta que los pacientes que previamente no conseguían una extensión activa de los dedos, con el tratamiento mejoran la posición pero raramente la función, y los pacientes con extensión activa de los dedos siempre se benefician funcionalmente del tratamiento.

En cuanto a las técnicas utilizadas, habría que señalar la idoneidad del grado de tensión aplicada al trasplante del tendón del cubital anterior (Beach, W. R. et al., 1991), suficiente para mantener pasivamente la muñeca en extensión neutra. Ello ha permitido también corregir la exagerada pronación que suelen presentar estas manos, sin necesidad de actuaciones complementarias, aunque puede también haber influido la relaja- ción proporcionada por la aponeurotomía de la musculatura epitroclear, como sugiere también otros autores (El-Said, M. S.,2001).

En las artrodesis de muñeca no se han utilizado placas. Si bien algunos autores consideran que dan una mayor estabilidad (Alexander, R.D. et al., 2000; Rayan, G.M. y Young, B.T., 1999), puede ser un inconveniente su prominencia en el dorso de la mano y la posibilidad de provocar adherencias en los tendones extensores, además de bloquear la fisis si estuviera aún abierta; tampoco hay que olvidar la frecuente osteoporosis, por inactividad, que suelen presentar algunas de estas manos, lo cual hace dudar que la fijación pueda ser estable (Hoffer, M. M. y Zeitzew, S., 1988). La corrección de la flexión palmar de la muñeca comporta una compresión que estabiliza la muñeca, y la introducción de la prominencia del hueso grande en la epífisis radial bloquea la posibilidad de un desplazamiento lateral; es por ello que han sido suficientes unas agujas de Kirschner si la fisis está abierta, o unas grapas dorsales, para impedir se reproduzca la flexión palmar de la muñeca, que en realidad mantiene ya corregida la presión del vendaje de yeso.

La resección de la hilera proximal del carpo ha facilitado la corrección de la flexión de la muñeca, como aceptan también otros autores (Alexander, R. D. et al., 2000; Rayan, G. M. y Young, B. T., 1999), y al acortar el esqueleto, facilita el balance muscular y también evita una excesiva distensión de las estructuras palmares.

\section{BIBLIOGRAFÍA}

Alexander, R. D.; Davis, J.R.; Peace, L.C.; Giddewal, M.A.: Wrist arthrodesis in children with cerebal palsy. J. Pediatr Orthop, 2000; 20: 490-495.

Beach, W. R.; Strecke, W. B.; Coe, J.; Manske, P.R. et al.: Use of the Green transfer in treatment of patients with spastic cerebral palsy: 17-year experience. J. Pediatr Orthop, 1991; 11: 731-736.

El-Said, N. S.: Selective release of the flexor origin with transfer of the flexor carpi ulnaris in cerebral palsy. J. Bone Joint Surg, 2001; 83: 259-262.

Hoffer, M.M.; Zeitzew, S.: Wrist fusion in cerebral palsy. J. Hand Surg, 1988; 13: 667-670.

Minguella, J.; Huguet, R.: Indicaciones y técnicas de la artrodesis de muñeca en la infancia y adolescencia. Rev. Esp. Cirg. Mano, 1979; 7: 63-74.

Rayan, G. M.; Young, B. T.: Arthrodesis of the spastic wrist. J. Hand Surg, 1999; 24 A: 944-952.
Sakellarides, H. T.; Kirvin, F. M.: Management of the unbalanced wrist in cerebral palsy by tendon transfer. Ann Plast Surg, 1995; 35: 90-94.

Wenner, S. M.; Johnson; K. A.: Transfer of the flexor carpi ulnaris to the radial wrist extensors in cerebral palsy. J. Hand Surg, 1988; 13: 231-233.

Wolf, T. M.; Clinkscales, C.M.; Hamlin, C.: Flexor carpi ulnaris transfer in cerebral palsy. J. Hand Surg, 1998, 23 B: 340-343. 